\title{
Nano-emulsions. Comprehensive Report
}

May 11, 2021

Steven P Allen, Richey M Davis, Eli Vlaisavljevich, Craig H Meyer

\section{Overview}

This report gives comprehensive review of the nano-emulsion project between UVA and Virginia Tech which has been generously supported by the Focused Ultrasound Foundation. The purpose of this project is to develop an acoustic coupling bath that is effectively invisible to MRI scans and also remains acoustically compatible with clinical transcranial FUS procedures. This remains important to the clinical translation of FUS because the commonly used acoustic coupling bath of degassed water persistently degrades the quality of MR guidance imaging. Our project proposes an acoustic coupling bath doped with specially designed iron oxide nanoparticles that 1) have a high ability to decay MRI signals, (as quantified by the $\mathrm{r}_{2}$ relaxivity), 2) have diameters less than $100 \mathrm{~nm}$, and 3) are coated with a hydrophilic coating. We hypothesize that these three innovations will produce a lightly doped water bath with identical mechanical, acoustic, thermal, biocompatibility, and electromagnetic properties as water, but with virtually no observable appearance or effect on guidance MR imaging. Finally, the low particle concentrations, small particle sizes, and specific coating will prevent the particles from stabilizing gas bubbles and seeding cavitation nuclei in the transducer's pre-focal field.

We are pleased to report nearly total success on this project, with an affirmation of all hypotheses stated above. The following table presents the milestones for this project, The rest of the report details our methods, results, and conclusions.

\begin{tabular}{|l|l|l|}
\hline \multicolumn{1}{|c|}{ Milestones } & \multicolumn{1}{c|}{ Deliverables } & \multicolumn{1}{c|}{ Status } \\
\hline $\begin{array}{l}\text { Nanoparticles formulated. } \\
\text { Relaxivity and cavitation } \\
\text { thresholds measured. } \\
\text { (Aims 1A, 1B) }\end{array}$ & $\begin{array}{l}\text { Deliverable 1: Report summarizing } \\
\text { nanoparticle cavitation threshold for } \\
\text { each sonication scheme, surface } \\
\text { coating, and concentration. Relaxivity } \\
\text { results. }\end{array}$ & Complete \\
\hline $\begin{array}{l}\text { In vitro estimation of } \\
\text { image quality in gel } \\
\text { targets (Aim 1C) }\end{array}$ & $\begin{array}{l}\text { Deliverable 2: Report including } \\
\text { thermometry error in gels when using } \\
\text { nanoparticle-doped water bath; RMSE } \\
\text { and SSi of gel images over time } \\
\text { following cessation of water } \\
\text { circulation; and static and transmit } \\
\text { magnetic field error. }\end{array}$ & Complete \\
\hline
\end{tabular}




\begin{tabular}{|l|l|l|}
\hline $\begin{array}{l}\text { In vivo validation study } \\
\text { complete (Aim 2B) }\end{array}$ & $\begin{array}{l}\text { Deliverable 4: Final comprehensive } \\
\text { report, including Q4 in-vivo imaging } \\
\text { results, uploaded to the FocUS Archive } \\
\text { (Foundation pre-print server). }\end{array}$ & Complete \\
\hline $\begin{array}{l}\text { Biocompatibility assessed } \\
\text { (Aim 2A) }\end{array}$ & $\begin{array}{l}\text { Deliverable 3: Report including } \\
\text { relative fluorescence microscopy } \\
\text { intensity of Alamar blue and CFDA- } \\
\text { AM dyed cell cultures, per exposure to } \\
\text { varying nanoparticle concentrations. }\end{array}$ & Complete \\
\hline $\begin{array}{l}\text { Project complete: } \\
\text { Publication ready } \\
\text { manuscript written } \\
\text { describing research and } \\
\text { results }\end{array}$ & $\begin{array}{l}\text { Deliverable 5: Submission of } \\
\text { publication in open-access journal. }\end{array}$ & In Progress. \\
\hline & $\begin{array}{l}\text { Deliverable 6: Submit abstract to the } \\
\text { next International Symposium on } \\
\text { Focused Ultrasound, the FUS } \\
\text { Foundation's biennial meeting. }\end{array}$ & Complete \\
\hline
\end{tabular}

\section{Overview of Particle Design}

In brief, An iron salt solution was prepared by dissolving iron(II) sulfate heptahydrate and iron(III) chloride hexahydrate at $170 \mathrm{mg} / \mathrm{mL}$ and $240 \mathrm{mg} / \mathrm{mL}$, respectively. The iron salts were placed in an ultrasonic bath (Model 15337410, S/N BXG021687330B, Fisher Scientific, Pittsburgh, PA) at room temperature for 1 hour to ensure complete dissolution. The iron salt solution was loaded into a $60 \mathrm{~mL}$ disposable syringe and connected to a confined impinging jet (2-jet) mixer ${ }^{1}$. The iron salt solution was injected into the 2-jet mixer against a stream of $17.5 \% \mathrm{w} / \mathrm{w}$ ammonia in water using a syringe pump (Harvard Apparatus PHD 4400 Programmable, Cat 70-202, S/N B-30980, Harvard Apparatus, Holliston, MA) at $0.5 \mathrm{~mL} / \mathrm{s}$ to achieve turbulent mixing conditions within the mixing chamber. The effluent from the mixer was immediately deposited into a flask containing a $7.36 \mathrm{mg} / \mathrm{mL}$ sodium poly(methacrylic acid) (PMAA) solution. The IBCM nanoparticle suspension was returned to the ultrasonic bath for 30 minutes then placed on a benchtop at room temperature overnight. The IBCM nanoparticles were washed and isolated with a combination of dialysis and centrifugal sedimentation. Dialysis was performed against ultrapure deionized water over 48-hours using cellulose-ester dialysis tubing (100 kDa, MWCO, 0867141, Fisher Scientific). The deionized water was replaced 5 times during the 48-hour period to ensure efficient removal of any free PMAA. Following dialysis, the IBCM nanoparticle suspension was split between 50-mL Eppendorf tubes and centrifuged (Sorvall Legend 
X1R, S/N 41067318, ThermoFisher Scientific, Waltham, MA) for 60 minutes at 20,000 rcf. The supernatant containing the desired IBCM nanoparticle population was collected, and the pellet was discarded.

The particles were found to be very monodisperse, with a mean hydrodynamic diameter of $31 \pm 1 \mathrm{~nm}$. The mean diameter of the particles varied by less than $10 \%$ after storage in a refrigerator for 90 days, suggesting that the particles remain highly stable and well dispersed throughout the medium.

(1) Han, J.; Zhu, Z. X.; Qian, H. T.; Wohl, A. R.; Beaman, C. J.; Hoye, T. R.; Macosko, C. W., A simple confined impingement jets mixer for flash nanoprecipitation. Journal of Pharmacentical Sciences

2012, 101 (10), 4018-4023.

\section{Relaxivity Estimates}

To measure the relaxivity of these particles, $10 \mathrm{~mL}$ of $0.9,0.45,0.225$, and $0.09 \mathrm{mM}$ Fe dilutions were stored in conical Eppendorf tubes. These were then placed in the bore of a 3T MRI scanner (MR 750, GE, Waukesha, WI) and subject to inversion recovery and multi-spin-echo sequences, which are the gold standard methods to estimate the T1 and T2 relaxation times of a given sample.

The sequences did a very good job of sampling both exponential decay curves for all but the T2 decay curve of the $0.09 \mathrm{mM}$ samples. All sampled relaxation curves were very well described by monoexponential decay. Some examples of the sampled data points (circles) and their corresponding least squares fits (lines) are shown below.
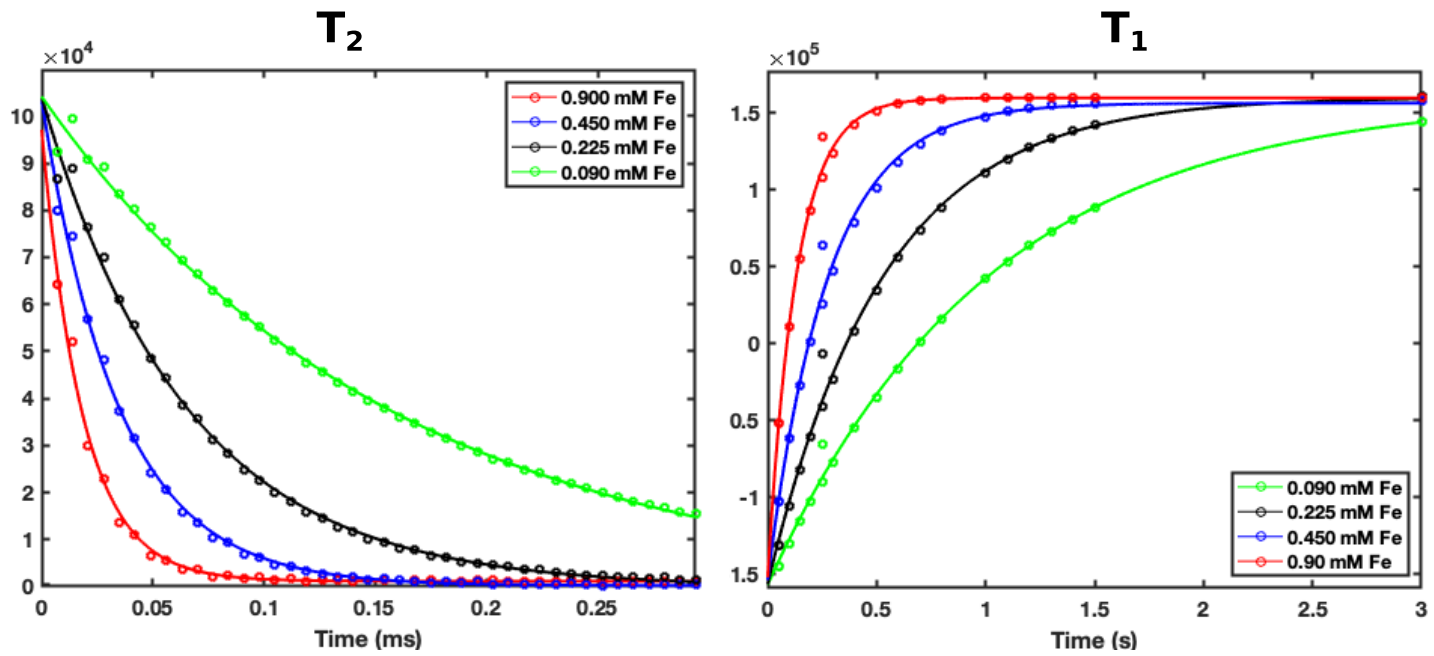
The characteristic decay times from each of these curves can be plotted as a function nanoparticle concentration. The slope of the resulting line is the relaxivity of the particles.
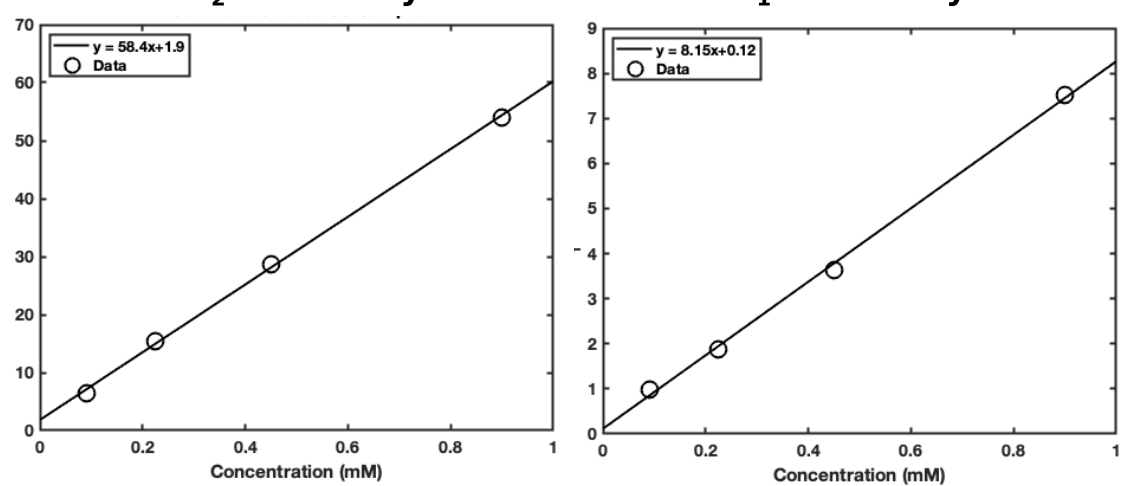

The relaxivity of these particles compared to commercial particles obtained during previous iteration of our project are also displayed in a table below. As can be observed, the particles generated in our lab possess a much smaller relaxivity than the commercial particles. This means that our particles will be less efficient than the commercial particles at suppressing the MR signal of the coupling bath.

Therefore higher concentration of the particles tested here will be required to achieve the same effects as those achieved with the commercial iteration.

The relatively smaller relaxivity is likely that the commercial particles would aggregate into clumps with a diameter about 7 times larger than the particles tested here. The larger the particle, or aggregation of particles, the larger the spatial extent of the magnetic field gradient the particle imposes as the magnetic field smoothly varies from the magnetic field strength at its surface (as calculated using the particle's magnetic susceptibility) and the ambient magnetic field strength. The field gradient, while microscopic, is what enables iron oxide to so efficiently accelerate $\mathrm{T}_{2} \mathrm{MRI}$ signal decay. Thus, larger particles tend to exhibit larger relaxivities and smaller particles, such as those discussed in this report, produce relatively smaller relaxivities. This results, is, therefore, not totally unexpected.

\begin{tabular}{|c|c|c|}
\hline Particle Source & $\mathbf{r}_{2}$ Relaxivity $\left(\mathbf{s}^{-1} \mathbf{m M}^{-1} \mathbf{F e}\right)$ & $\mathbf{r}_{1}$ Relaxivity $\left(\mathbf{s}^{-1} \mathbf{m M}^{-1} \mathbf{F e}\right)$ \\
\hline $\begin{array}{c}\text { Previous Iteration, } 240 \mathrm{~nm} \\
\text { diameter, Commercial } \\
\text { Source }\end{array}$ & 175 & Not Measured \\
\hline $\begin{array}{c}\text { Current Iteration, } 31 \mathrm{~nm} \\
\text { diameter }\end{array}$ & $58.40 \pm 0.03$ & $8.19 \pm 0.15$ \\
\hline
\end{tabular}

\section{Pulsed cavitation threshold results}

Insonating a sample with low duty-cycle, single acoustic pulses remains one of the best methods to understand the cavitation threshold pressure for a given acoustic frequency. The same dilutions tested during the relaxivity experiments described above were also subjected to single-cycle acoustic cavitation tests. These experiments yielded an estimate of the probability of cavitation as a function of peak negative acoustic pressure. Our results indicated that the 0.5 cavitation probability threshold pressure remained invariant with nanoparticle concentration. However, for the $0.9 \mathrm{mM}$ concentration (labeled 
"High Concentration" in the figure below), non-trivial cavitation probabilities occurred at pressures below the 0.5 probability threshold pressure. These results suggest that some care must be taken to ensure that the prefocal acoustic field does not reach these peak negative pressures.

Degassed solutions of 0.9, 0.09, and $0.009 \mathrm{mM}$ Fe concentrations were placed in a chamber at the focused of $500 \mathrm{kHz}$, intrinsic threshold, histotripsy transducer. Passive acoustic detectors and a camera were used to record cavitation events, which were then tabulated. The relative fraction of acoustic pulses yielding a cavitation event at a particular peak negative pressure was labeled the "probability of cavitation." The results are displayed in the following figure.

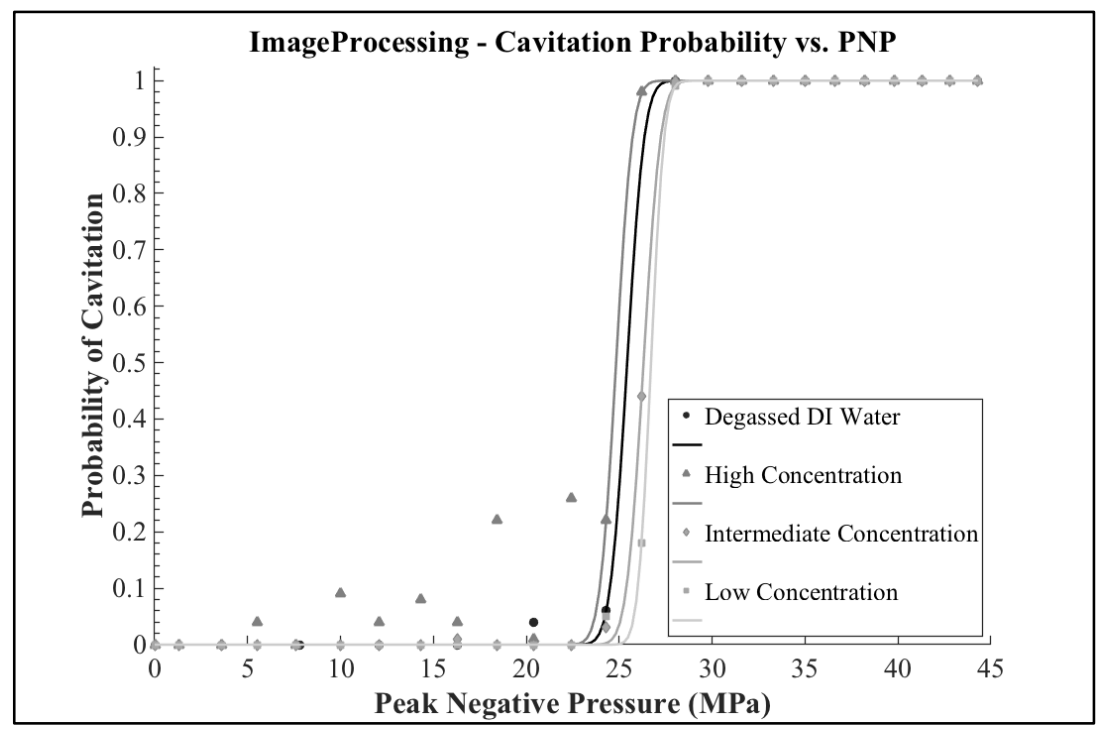

Cavitation threshold data, derived from the figure above, are presented in this following table. It appears that the 0.5 cavitation probability threshold pressure, $\mathrm{p}_{\mathrm{t}}$, is invariant with particle concentration over the concentration ranges tested here. $\mathrm{p}_{\min }$ refers to the minimum pressure at which a non-zero cavitation probability was observed. The term $\sigma$ is a measure of how gradually the threshold curve appears to increase with negative pressure.

\begin{tabular}{|l|l|l|l|}
\hline Sample & $\begin{array}{l}p_{t} \\
\mathrm{MPa}\end{array}$ & $\begin{array}{l}p_{\text {min }} \\
\mathrm{MPa}\end{array}$ & $\sigma$ \\
\hline Degassed DI Water & 25.4 & 20.4 & 0.72 \\
\hline $0.9 \mathrm{mM} \mathrm{Fe}$ & 24.8 & 5.5 & 0.69 \\
\hline $0.09 \mathrm{mM} \mathrm{Fe}$ & 26.3 & 16.3 & 0.72 \\
\hline $0.009 \mathrm{mM} \mathrm{Fe}$ & 26.7 & 24.3 & 0.56 \\
\hline
\end{tabular}




\section{Continuous wave cavitation threshold results}

The same particles, with the same concentrations, were subjected to continuous wave cavitation experiments. These are a modification of the pulsed experiments described above where the particles in the chamber are insonated for 10s using a continuous wave acoustic pulse at $650 \mathrm{kHz}$. Passive acoustic detectors then record the relative fraction of the 10 s insonation where cavitation events are thought to occur. These curves, while not equivalent to a "probability of cavitation," also demonstrate a sigmoidal shape and can suggest the frequency of cavitation events occurring at a given peak negative pressure. Our results are shown below.

To better capture statistical variability, this set of experiments were run on two independently performed dilutions: one diluted at Virginia Tech (labeled 'T"), and one diluted at UVA (labeled "U"). The resulting sigmoidal curves are displayed below.

"T". Source

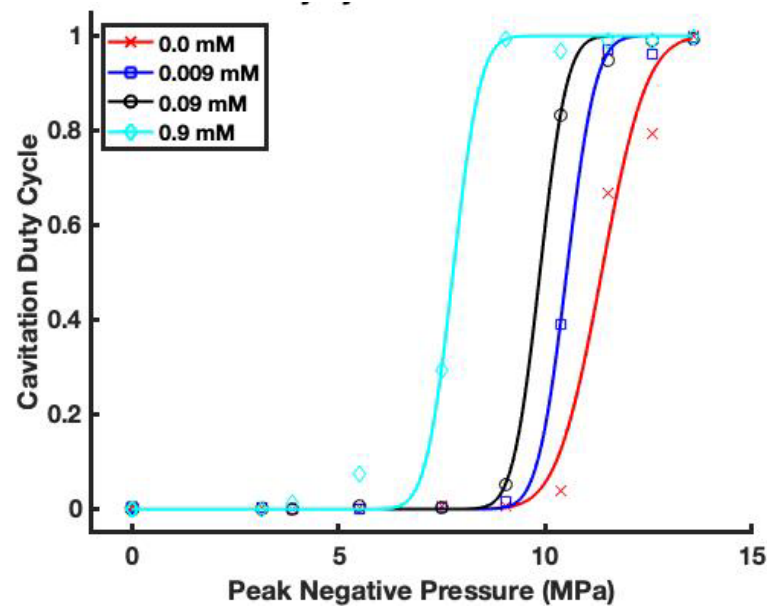

"U" Source

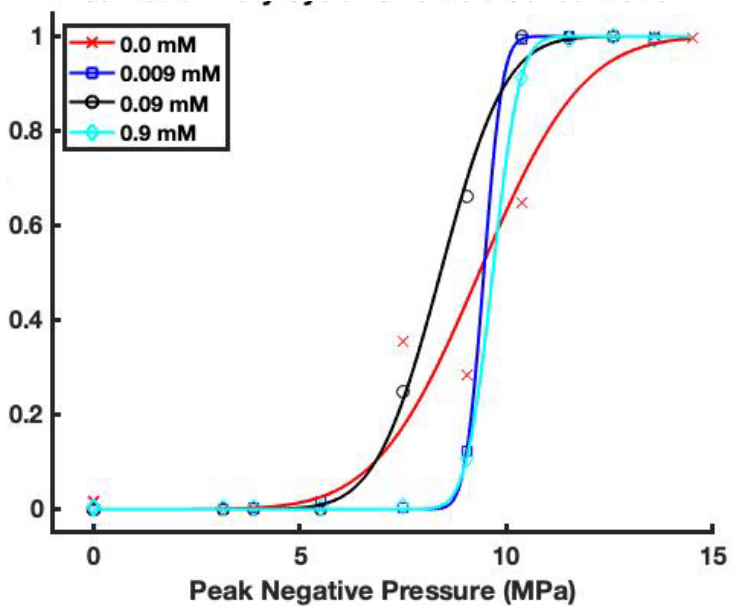

Save for one important outlier (the $0.9 \mathrm{mM}$ Fe sample from source "T"), the curves represent very similar 0.5 cavitation duty cycle threshold pressures. In the case of the $0.9 \mathrm{mM}$ sample from source "T," experimental error allowed some dissolved oxygen (and likely other gases) to enter the solution prior to testing. Combined with the more gradual slopes observed for some samples derived from source "U," it is still unclear if the cavitation behavior is being determined by the concentration of nanoparticles in solution or gas content or both. To further clarify this point, we plot the 0.5 cavitation duty cycle threshold pressures as both a function iron concentration and measured dissolved oxygen content below. 

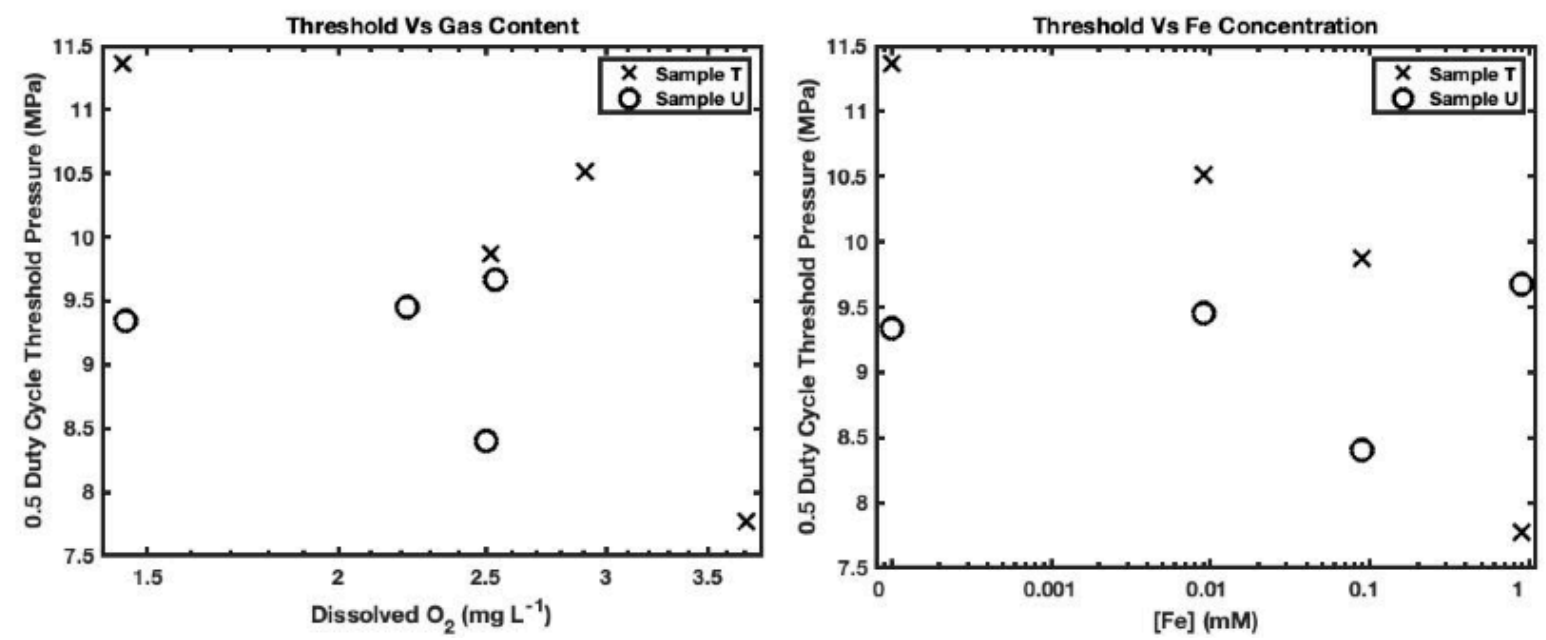

As can be seen in the above figure, the threshold appears to decrease slightly with particle concentration and gas content. However, it is still unclear to our group which of the differences in the thresholds displayed in these figures are statistically significant and the exact relationship is difficult to determine from the data at hand. However, this behavior is very different from that observed using the commercially available particles from our previous iteration. For those particles, the threshold pressure (as measured using high duty cycle insonations) decreased monotonically with acoustic pressure as displayed below in a figure copied from a previous publication of ours:

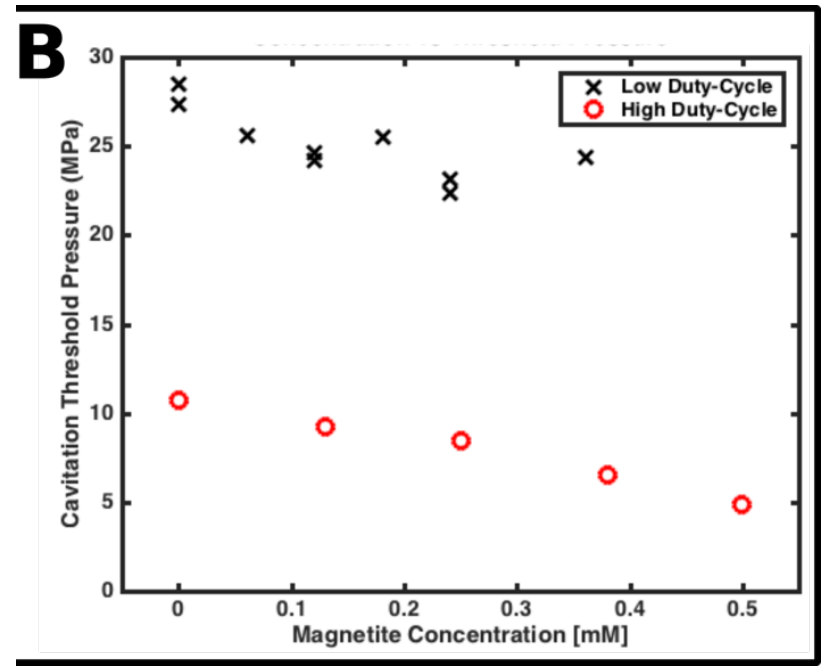

\section{In Vitro Estimation of Image Quality.}

\section{Introduction}

This section reports our team's efforts the examine the following hypotheses about guidance imaging quality

1. The nano-emulsion will remove guidance imaging artifacts induced by water motion undergoing "pulsatile" motion as experience in clinical tcMRgFUS procedures. 
2. The nano-emulsion will permit continuous water circulation during guidance MR imaging.

\section{Methods}

These hypotheses were evaluated by conducting guidance MR imaging scans during a sham tcMRgFUS thalamotomy procedure on a gel phantom. Briefly, an agar phantom was placed in a 1024 phased array transducer (Exablate Neuro, INSIGHTEC, Haifa, Israel). Using a research-dedicated water pump and degassing system, the space between the phantom and the transducer surface was filled with either degassed water or a degassed nano-emulsion sample with a relaxation rate between 170 and $200 \mathrm{~s}^{-1}$ or, equivalently, a T2 relaxation time between $5-6 \mathrm{~ms}$. The true relaxation time of the sample could not be determined with greater precision than that reported above because samples with very large relaxation rates produce larger uncertainties in the relaxation rate estimation method.

Images of the phantom were acquired using the following three sequences

\begin{tabular}{|c|c|c|}
\hline Sequence type & Guidance Purpose & Relevant Parameters \\
\hline Turbo-Spin Echo & $\begin{array}{l}\text { Anatomic reference, evidence } \\
\text { of lesioning }\end{array}$ & $\begin{array}{l}\text { TR: } 2.5 \text { s; TE: } 101 \mathrm{~ms} \text {; ETL: } \\
\text { 16; FOV: } 28 \mathrm{~cm} \text {; Resolution: } \\
\text { 1.1 x } 2.2 \text { x } 3 \text { mm; Pixel } \\
\text { Bandwidth: } 122 \mathrm{~Hz}\end{array}$ \\
\hline MP-RAGE & $\begin{array}{l}\text { Anatomic Reference, } \\
\text { Membrane Fold Detection }\end{array}$ & $\begin{array}{l}\text { TR: } 5.5 \mathrm{~s} \text {, TE: } 1.9 \mathrm{~ms} \text {; TI: } 450 \\
\text { ms; FOV: } 28 \mathrm{~cm} \text {; Resolution: } \\
\text { 2.2x2.2x3 mm; Pixel } \\
\text { Bandwidth: } 244 \mathrm{~Hz} .\end{array}$ \\
\hline Spoiled-GRE & Thermometry & $\begin{array}{l}\text { TR: } 34 \mathrm{~ms} \text {; TE: }[3.3,8.1,12.8 \text {, } \\
\text { 17.6, 22.4, } 27.1 \mathrm{~ms}] \text {; FOV: } 28 \\
\text { cm; Resolution: } 1.1 \times 2.2 \times 3 \\
\text { mm; Pixel Bandwidth: } 278 \mathrm{~Hz}\end{array}$ \\
\hline
\end{tabular}

These sequences were used to acquired images under the following motion conditions for both fluid states (degassed water and the degassed nano-emulsion)

\begin{tabular}{|c|c|}
\hline Fluid Motion State & Fluids Tested \\
\hline Stationary & Water, Nano-emulsion \\
\hline Pulsatile & Water, Nano-emulsion \\
\hline Continuous & Water, Nano-emulsion \\
\hline
\end{tabular}

Where "pulsatile" refers to the clinically likely case of continuous fluid circulation for 2 minutes before turning the circulation pump off at the onset of image acquisition. "Stationary" refers to allowing the fluid volume to dissipate momentum for 10 minutes prior to image acquisition.

The effects of water motion were evaluated in the acquired TSE images by comparing the root mean square error (RMSE) between pixels within a region of interest inside the gel phantom acquired during the stationary motion state and those within the same region of interest acquired in the other two motion states. Briefly, a region within the phantom was selected via an Otsu thresholding operation on the scan acquired with the nano-emulsion in a stationary state. Holes within the thresholded region were filled and the edges of the selected object were eroded 120 pixels to avoid edge effects. Because 
the phantom was able to rotate and shift slightly ( $<2$ degrees and $<1 \mathrm{~mm}$ ) between scans, this ROI was then rotated and translated to match phantom geometry of all other acquired TSE scans. Assuming that the gel is homogeneously well mixed and phantom movement between scans is small, the magnitude of registered pixels within the ROI between each scan should deviate only from the effects of acquisition noise and fluid motion.

The effects of water motion were evaluated in the acquired spoiled GRE images by first computing the estimated changes in temperature within the phantoms using the optimized temperature reconstruction described in Odeen et al and then computing the through-time standard deviation of the resulting

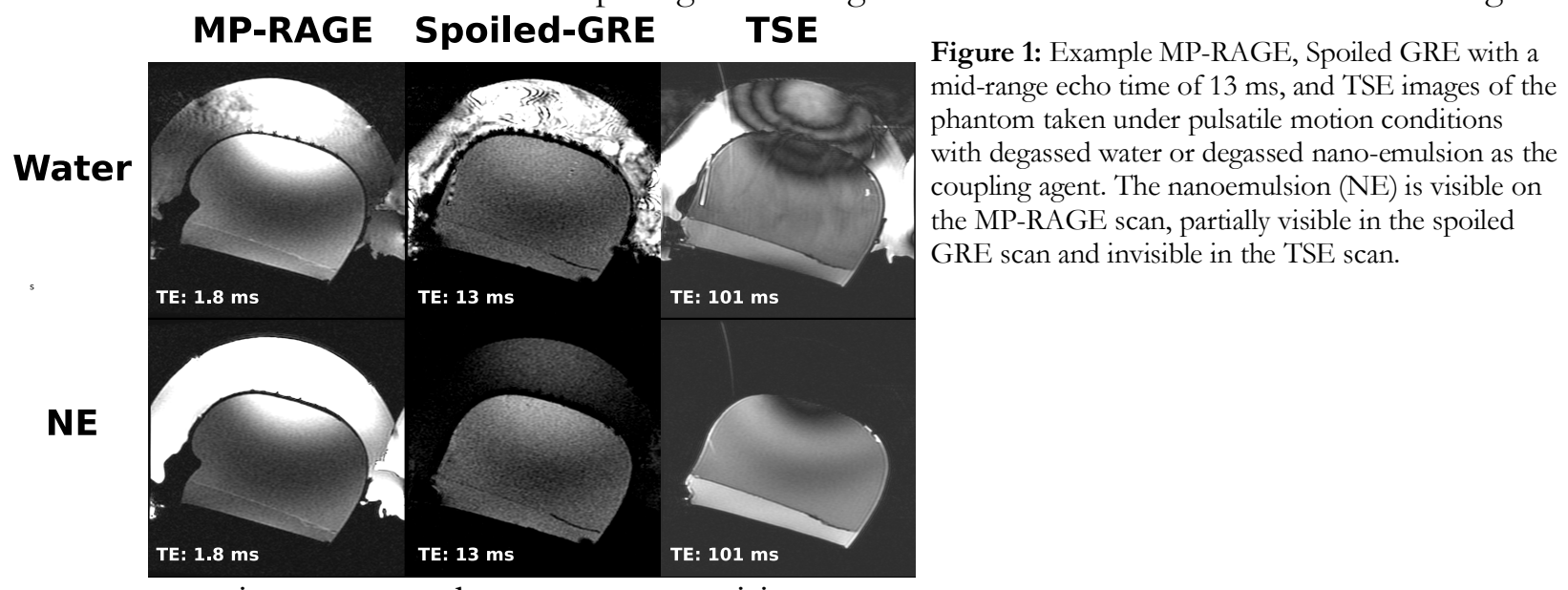

temperature estimates, termed temperature precision.

\section{Reference:}

Odéen, Henrik, and Dennis L Parker. 2019. “Improved MR Thermometry for Laser Interstitial Thermotherapy." Lasers in Surgery and Medicine 51 (3): 286-300.

\section{Qualitative Results.}

When acquired with water as a coupling agent, all scans showed strong fluid signal as well as artifacts from water motion. When acquired with the nano-emulsion as a coupling agent, only MPRAGE scans and the spoiled GRE scans acquired with echo times at 3.3 and $8.1 \mathrm{~ms}$ displayed strong fluid signal and motion artifacts. Meanwhile, all TSE scans and spoiled GRE scans with later echo times displayed greatly reduced to negligible fluid signal. Example images acquired in the "pulsatile" motion state are shown in Figure 1.

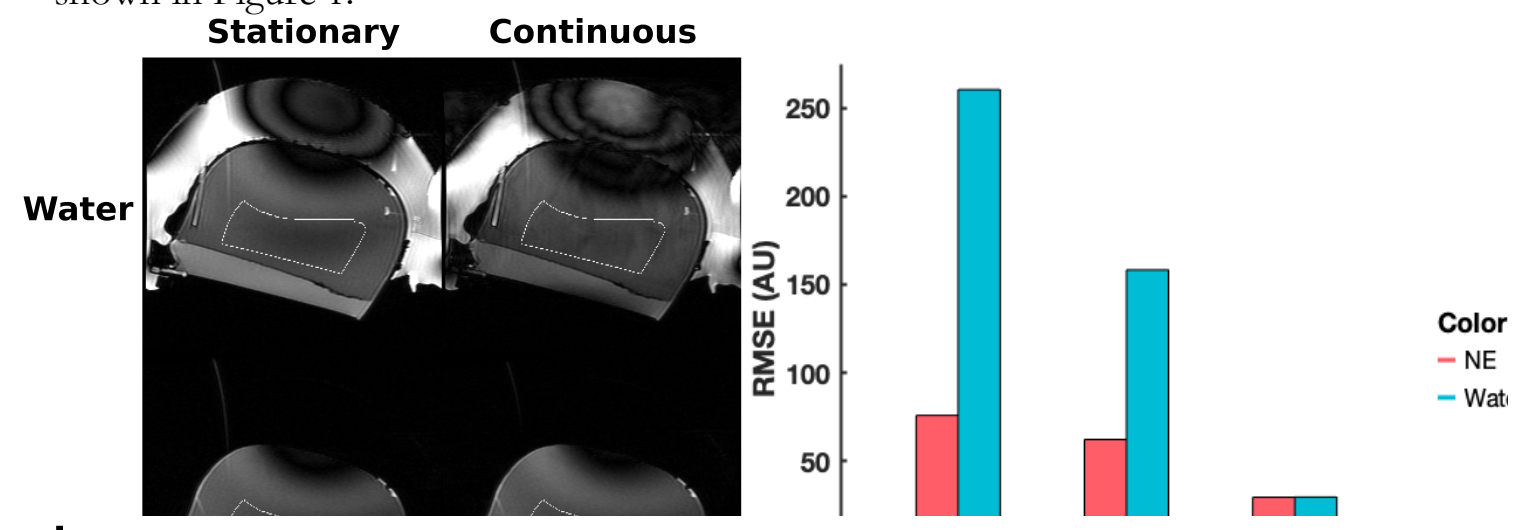

IFigure 2: (Left) Example TSE images acquired in the stationary and continuous motion states for both the water and nano-emulsion (NE) coupling fluids. Scans with the nano-emulsions in the continuous state show a dramatic decrease in RMSE. The ROI used for motion analysis is displayed as well. (Right) RMSE between the stationary and two other motion states computed within the ROI displaved at left. 


\section{Quantitative Results}

Figure 2 displays example TSE images acquired with the coupling fluid in stationary and continuous motion states alongside RMSE errors found within the phantom during all examined motion states. The nano-emulsion greatly reduces differences in pixel magnitude between the stationary motion state and the pulsatile and continuous motion states. Some residual error remains-which could stem from residual motion artifacts or changes in transmit field shading.

Figure 3 displays the temperature precision found in the phantom for all motion states and fluid compositions tested in this report. In both periodic and stationary motion states, the phantom displayed a temperature precision between $0.5-1.5^{\circ} \mathrm{C}$ regardless of the coupling fluid. However, continuous water circulation introduced time-dependent image artifacts that decreased temperature precision to $1-2{ }^{\circ} \mathrm{C}$ in some regions of the phantom. The use of the nano-emulsion returned temperature precision back to a range of $0.5-1.5^{\circ} \mathrm{C}$.

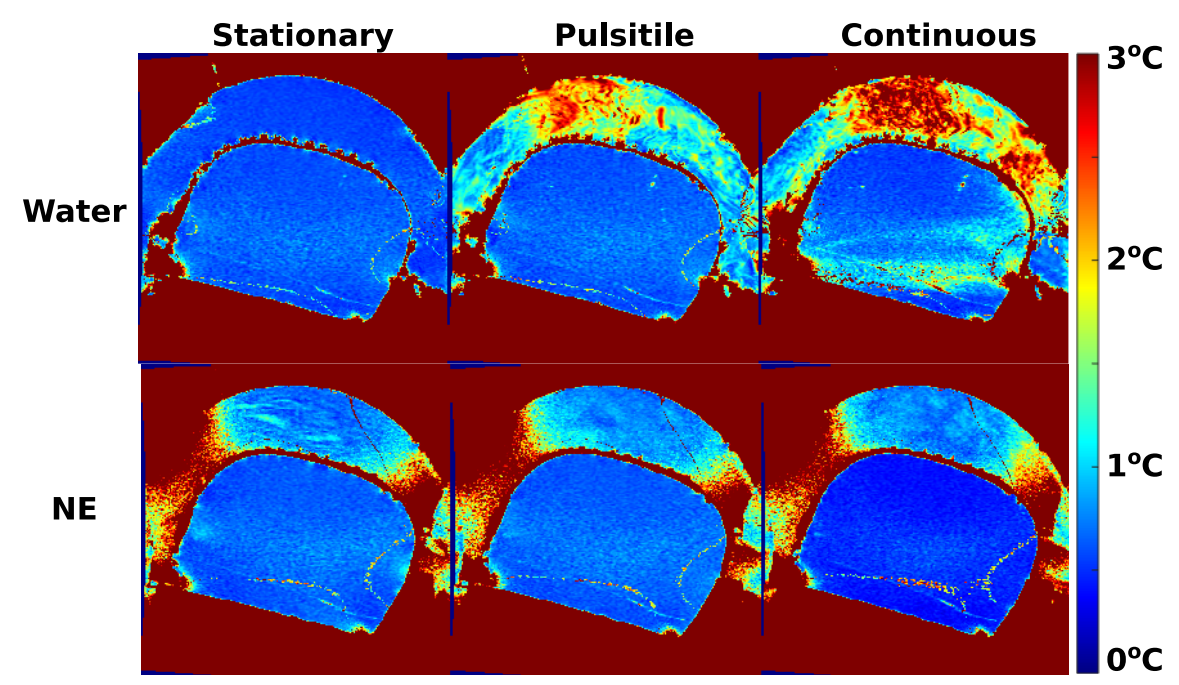

Figure 3: A map the through-time standard deviations of temperature estimates made in a phantom for all motion states and fluid types. For all motion states, the use of the nanoemulsion (NE) does not adversely affect thermometry precision relative to that of water. In the case of continuous fluid circulation, the nanoemulsions remove motion-based artifacts and improve temperature precision relative to that of water.

\section{Trans-Skull Sonication}

\section{Introduction}

This section reports our team's efforts the examine the following hypotheses about guidance imaging quality

1. The nano-emulsion will not impede acoustic transmission at high powers.

2. The nano-emulsion will not stimulate cavitation activity in the pre-focal field

\section{Methods}

To test the above hypotheses, the cranium of a cadaver skull was filled with acoustically absorptive gel, mounted on a support frame, and placed at the focus of a $650 \mathrm{kHz}, 1024$ phased array transducer (Exablate Neuro, INSIGHTEC, Haifa, Israel). The research-dedicated water pump and degassing system filled the space between the phantom and the transducer surface was filled with either degassed water or the degassed nano-emulsion sample. The dissolved oxygen contents of both fluids were maintained below $1.8 \mathrm{mg} / \mathrm{L}$ throughout the experiment. For each coupling fluid type, the gel was insonated four times at $1050 \mathrm{~W}$ acoustic power for 25 seconds. MR thermometry, consisting of the spoiled GRE sequence described above, occurred simultaneously. All insonations were prescribed to 
the same coordinates. Finally, in order to maximize acoustic pressure in the prefocal field without producing temperatures above the melting point of the gel, the phased array was not adjusted to compensate for skull-induced phase-shifts. To maintain fidelity to clinical conditions, both fluids were maintained in the "pulsatile" motion state where the circulation pump was switched off at the onset of MRI acquisition. After insonation, was phantom was allowed to cool for 11 minutes while the coupling fluid underwent continuous circulation and degassing. The coupling fluid was not chilled.

Thermometry reconstructions were computed using the methods described above with the addition of using zero-padding to reconstruct the temperature information at a spatial resolution of $1.1 \times 1.1 \times 3$ $\mathrm{mm}$.

\section{Results}

For all 8 insonations, the clinical system reported stable cavitation emissions. However, these emissions neither increased in intensity between the water and nano-emulsion coupling fluid, nor triggered the clinical system to stop or modify the transducer's power output, as it is programmed to do in cases of high energy cavitation emissions. Upon closer inspection, the phantom hosted several stable bubbles that could not be entirely removed. It is likely that these are the sources of the cavitation emissions.

Figures $4 \mathrm{~A}$ and 4B display an example temperature map obtained during insonation using the nanoemulsion coupling bath along with temperature time courses of the hottest pixel within the focal target for all eight insonations. The time courses show excellent agreement regardless of the coupling fluid. This fact is further elucidated in frame $\mathrm{C}$ of Figure 4, which displays a Bland-Altman plot of the average temperature difference between 11 co-registered pixels within the treatment focus. The average difference in reported temperature was found to be $0.1{ }^{\circ} \mathrm{C}$ with a standard deviation of differences of $0.5^{\circ} \mathrm{C}$. The excellent agreement between the two coupling fluids indicate that the nano-emulsion presents an acoustically compatible coupling medium.

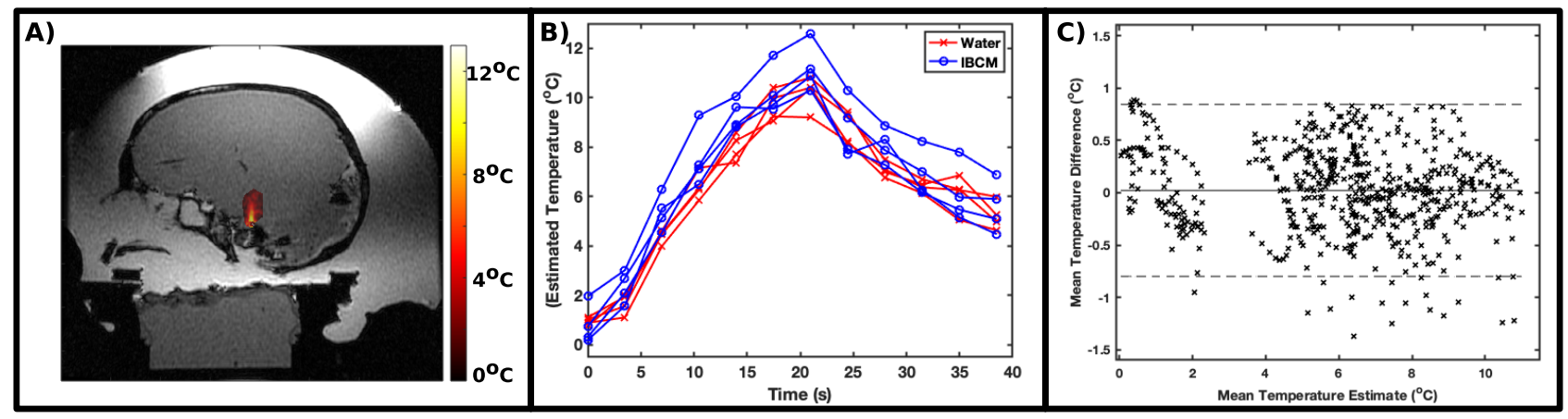

Figure 4: (A) Thermometry heat map superimposed on an image of the skull phantom embedded with acoustically absorptive gel. (B) Time courses from the hottest voxel across all 8 insonations. Temperature time courses remain quite similar regardless of the coupling material. (C) Bland-Altman plot of the temperatures recorded at 11 co-registered pixels for both coupling materials. Temperature differences display a standard deviation of $0.5^{\circ} \mathrm{C}$ and a negligible mean difference.

\section{In Vivo Imaging}

\section{Introduction}

This section reports our team's efforts the examine the following hypotheses about guidance imaging quality

1. The nano-emulsion will improve guidance MR image quality during a sham tcMRgFUS procedure in a human subject. 
2. The nano-emulsion will permit a smaller imaging field-of-view than currently implemented in clinical tcMRgFUS without adverse aliasing artifacts.

\section{Methods}

To test the above hypotheses, and with IRB approval, a human subject was fitted with a swim cap and placed at the focus of a $650 \mathrm{kHz}, 1024$ phased array transducer (Exablate Neuro, INSIGHTEC, Haifa, Israel) where the transducer's amplifier system was disconnected from the transducer. The space between the subject and the transducer surface was sequentially filled with degassed water and the nano-emulsion sample. The subject was then scanned with the TSE and spoiled GRE sequences described above. These scans were then repeated with a modified field of view of $18 \mathrm{~cm}$. For all scans, the fluids were kept in a "pulsatile" motion state.

\section{Results}

Figure 5 displays TSE images acquired of the human subject for both fields-of-view. The use of the nano-emulsion produces a marked reduction in motion and aliasing artifacts. For example, subtle grey/white matter differences are better seen using the nano-emulsion (blue arrows). Some aliased objects remain and were caused by residual water resting in water lines belonging to the clinical and circulation degassing system (red arrows). The roll-off in image intensity for all cases are caused by a standing wave RF effect that is beyond the scope of this project.

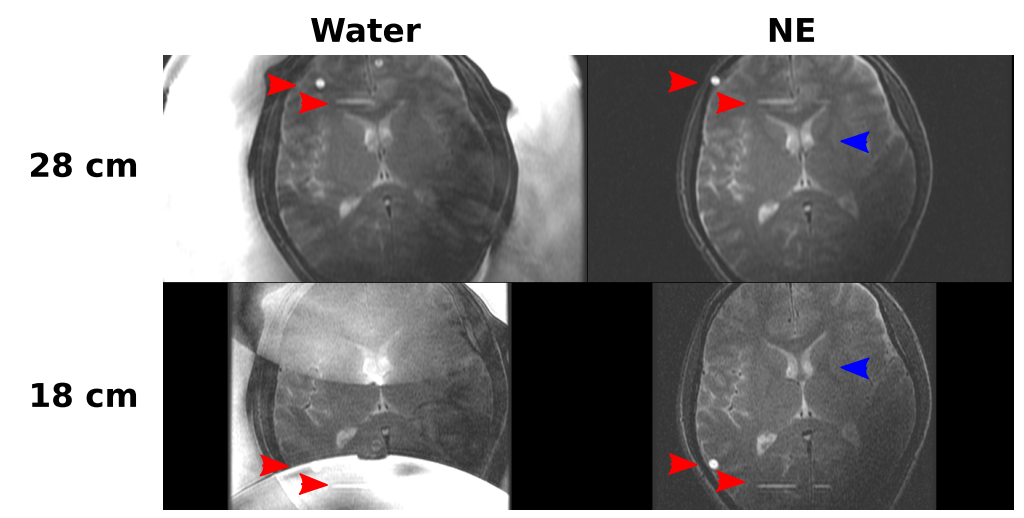

Figure5: TSE images acquired at 28 and $18 \mathrm{~cm}$ fields of view using both water and the nano-emulsion (NE) as an acoustic coupling agent. The nano-emulsion improves removes aliasing and motion artifacts and improves subtle grey/white matter contrast (blue arrows). Residual aliasing artifacts (red arrows) are caused by water resting in circulation lines used for clinical treatments.

Figure 6 displays temperature precision maps of the same human subject. The standard deviations reported in the $18 \mathrm{~cm}$ field-of-view cases have been artificially amplified by a factor of $(28 / 18)^{2}$ to compensate for the reduction in signal-to-noise imposed a the smaller voxel size. The figure shows

Figure 6: Maps of the through-time standard deviation of temperature estimates in a human subjects scanned with fields of view of 28 and $18 \mathrm{~cm}$ with water and the nano-emulsion (NE) as an acoustic coupling agent. All fluids were maintained in a "pulsatile" motion state. Like the phantom scans, the use of the nano-emulsion does not adversely affect the precision of the temperature estimate. However, some aliasing persists in the $18 \mathrm{~cm}$ scan due to the nano-emulsions's strong signal at the echo obtained at $3.3 \mathrm{~ms}$. 
that, like the results from the phantom shown above, the use of nano-emulsions does not decrease the precision of MR thermometry.

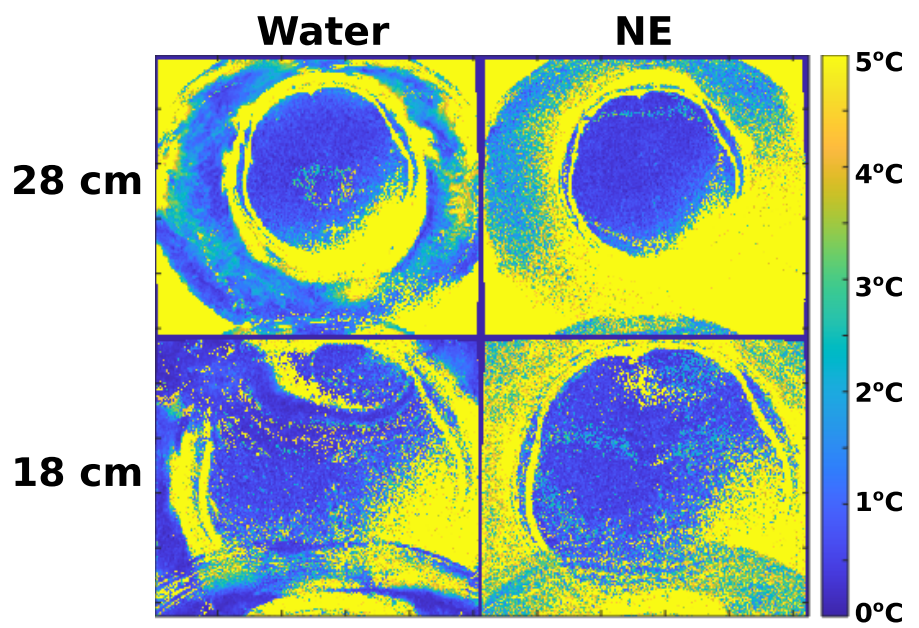

Finally, due to the bright appearance of the nano-emulsion in images acquired at 3.3 and $8.1 \mathrm{~ms}$, some aliasing artifact can be observed in the $18 \mathrm{~cm}$ field-of-view image even when using the nano-emulsion. However, these artifacts can be very easily avoided with almost no penalty by omitting data obtained at these two echoes from the temperature reconstruction. The short echo-times of these two data points relative to the long T2* times of white and grey matter ensure that they contain almost zero useful temperature information relative to those obtained at later echoes. Figure 7 displays temperature precision maps calculated identically to those in Figure 6 but with data acquired at 3.3 and $8.1 \mathrm{~ms}$ data omitted. The figures show identical temperature precision to that in Figure 6 without aliasing artifacts in the $18 \mathrm{~cm}$ field-of-view scans acquired with the nano-emulsion.

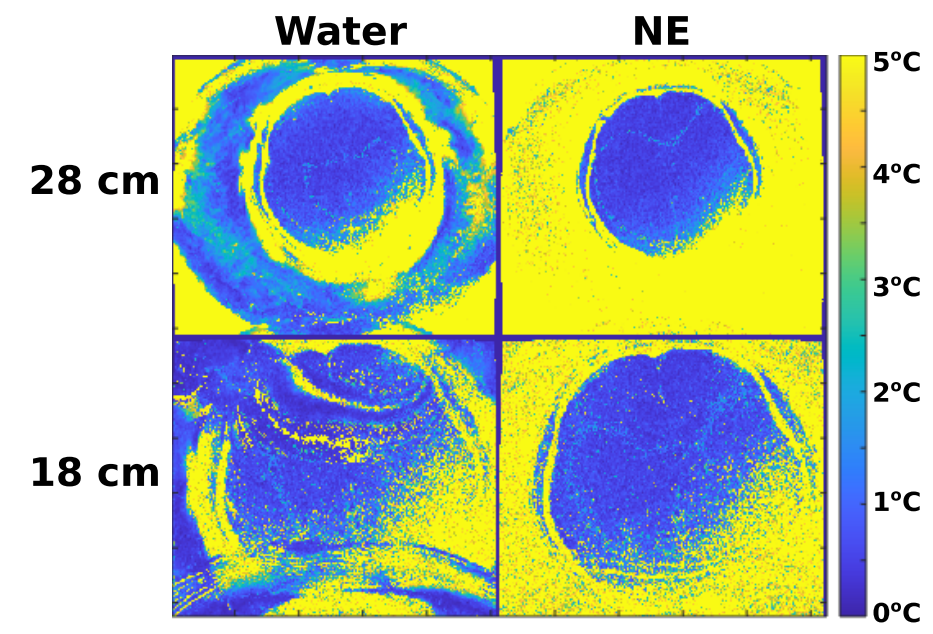

Figure 7: Maps of the through-time standard deviation of temperature estimates in a human subjects scanned with fields of view of 28 and $18 \mathrm{~cm}$ with water and the nano-emulsion (NE) as an acoustic coupling agent. All fluids were maintained in a "pulsatile" motion state. Temperature maps were reconstructed after omitting data acquired from the 3.3 and 8.1 ms echoes. Due to the low temperature information content of these echoes, aliasing and motion artifact from the coupling bath can be removed without penalty to temperature precision.

\section{In Vitro Biocompatibility Assay.}

\section{Introduction}

This section reports our team's efforts the examine the following hypotheses about our designed coupling bath

1. The nano-emulsion will not produce discernible cytotoxic or other adverse effects on human derived cell cultures. 


\section{Methods}

Human dermal fibroblasts (HDF) were seeded in cell culture wells at $\sim 4 \times 10^{\wedge} 4$ cells/well $(\sim 80 \%$ confluent in a 96-well plate) and incubated at $37{ }^{\circ} \mathrm{C}$ with $5 \% \mathrm{CO} 2$ overnight to allow for adherence. Wells were then assigned different exposure levels to the designed nanoparticles. Exposure parameters were nano-emulsion concentration $(0.000,0.009,0.09 \mathrm{mM}, 0.9 \mathrm{mM}, 3.75 \mathrm{mM} \mathrm{Fe})$ and exposure period ( $3 \mathrm{hrs}, 12 \mathrm{hrs}, 24 \mathrm{hrs}, 48 \mathrm{hrs}$ ). All experiments were conducted in triplicate. At each time point, the cells were washed with PBS and then incubated at room temperature for 15 minutes with the live/dead dyes (calcein AM and BOBO-3 Iodide). After staining, the cells were imaged using fluorescence microscopy.

As a negative control, a separate well was exposed to 5\% Triton X-100, which is known to be fatal to HDF.

\section{Qualitative Results.}

While the unexpected high cell density of the well plates make it difficult to count the relative ratio of live/dead cells after exposure, the microscopy images captured suggest almost complete HDF viability

$3 \mathbf{H r}$

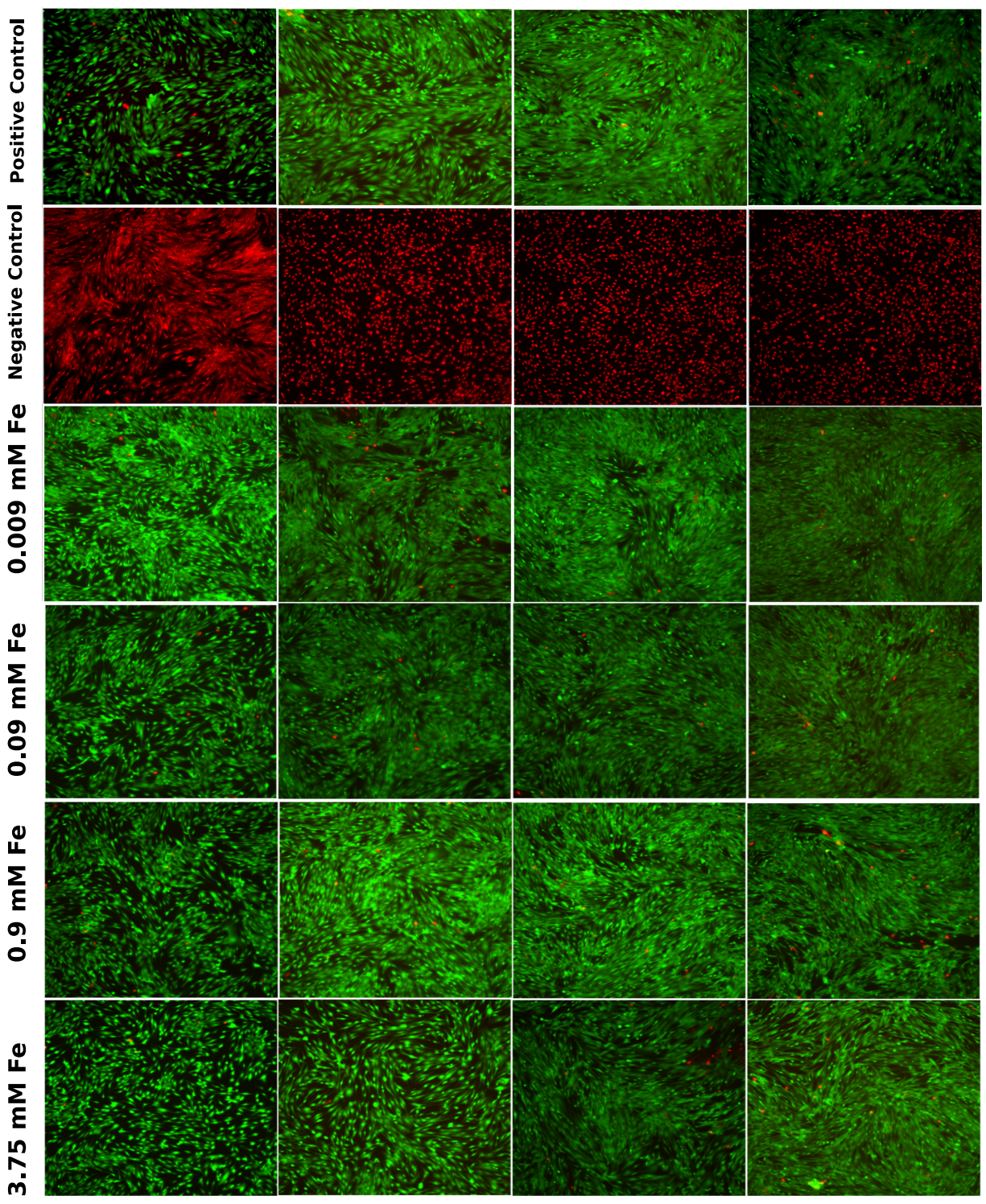


at all exposure levels. Example images of all exposure levels are shown in Figure 1. Live cells appear as green and dead cells appear as red.

\section{Summary}

This report demonstrate that our team has achieved all major goals of our study: the creation of an acoustically and biologically compatible coupling bath that greatly reduces guidance MRI artifacts relative to the use of degassed water. We are pleased with these results and grateful for the Foundation's support and guidance, without which this project would not be possible. 